\title{
NOTES
}

\section{Cross-sectional Morphologies of Polystyrene Nanospheres with Hydrophilic Corona Surfaces}

\author{
Takeshi Serizawa,,${ }^{1, \dagger}$ Yoshinori Yanagisono, ${ }^{1}$ Masamichi Ueno, ${ }^{2}$ \\ and Mitsuru AKASHI ${ }^{1, \dagger, \dagger \dagger \dagger}$ \\ ${ }^{1}$ Department of Nanostructured and Advanced Materials, Kagoshima University, \\ 1-21-40, Korimoto, Kagoshima 890-0065, Japan \\ ${ }^{2}$ Japan Immunoresearch Laboratories, 351-1 Nishiyokote-cho, Takasaki 370-0021, Japan
}

(Received April 12, 2004; Accepted October 21, 2004; Published January 15, 2005)

\author{
KEY WORDS Core-Corona Nanosphere / Cross-Section / Morphology / Transmission Electron \\ Microscope / Macromonomer / \\ [DOI 10.1295/polymj.37.39]
}

Polymeric nanospheres have potential applications in technological and biotechnological fields. It is important to control the nanosphere structures (e.g., sizes, size distributions, surface functionalities, and chemical compositions), followed by the development of new polymerization system. We have already developed a synthetic method for polymeric nanospheres, based on free radical copolymerization of hydrophobic monomers (styrene and methyl methacrylate) and hydrophilic macromonomers in polar organic solvents. ${ }^{1-3}$ During polymerization, amphiphilic graft polymers assemble in solution to form corecorona nanospheres, which have hydrophobic cores and hydrophilic corona layers on their surfaces, resulting in excellent aqueous phase dispersion. Surface functionalities (neutral, ${ }^{1-3}$ cationic, ${ }^{4,5}$ anionic, ${ }^{5,6}$ thermoresponsive units 7,8 ) can be readily modified by changing the macromonomer, and nanosphere size (e.g., $50 \mathrm{~nm}$ to $1 \mu \mathrm{m}$ ) can also be controlled by changing polymerization conditions. ${ }^{9-17}$ Furthermore, size distribution is narrow in all cases. Using functional groups introduced on corona, proteins, ${ }^{18}$ peptides, ${ }^{19}$ saccharides, ${ }^{20}$ and metal nanoparticles ${ }^{21}$ were conjugated on nanosphere surfaces, and their potential functions were also demonstrated. It is therefore important to understand surface and internal structures of nanospheres on the basis of physicochemical analyses, because functional molecules conjugated are potentially located on coronas.

In our previous study, cross-sectional structures of nanospheres with polystyrene (PSt) cores and poly(ethylene glycol) (PEG) coronas were analyzed by transmission electron microscope (TEM) observations. ${ }^{22}$ The core and corona could be visualized by exposure of nanosphere cross-sections in an osmium oxide atmosphere, resulting in TEM images with clear black and white contrasts for respective corona (increased staining) and core. Although PEG coronas are representative simply due to well-defined chemical structures and neutral charges, it is further attractive to analyze nanosphere structures with other coronas. In the present study, we analyzed the internal structures of nanospheres with PSt cores and two types of corona layers. One polymer chain used for the corona was poly(methacrylic acid) (PMAA) with chargeable carboxyl groups, and the other was poly( $N$-isopropylacrylamide) (PNIPAAm) with wellknown thermoresponsive properties. Since the former nanosphere has been well studied in the conjugation of proteins, ${ }^{18}$ and particle size of the latter decreases above a lower critical solution temperature (LCST) due to deswelling of PNIPAAm chain water molecules, ${ }^{23}$ the present study furthers our understanding of these physical properties.

\section{EXPERIMENTAL}

\section{Materials}

Core-corona nanospheres were prepared by free radical copolymerization of $\mathrm{St}$ and $p$-chloromethylstyrene-terminated macromomers of $\mathrm{MAA}^{5}$ or NIPAAm $^{10}$ in $5 \mathrm{~mL}$ of ethanol/water $(4 / 1, v / v)$ or $(7 / 3, v / v)$ in the presence of $2,2^{\prime}$-azobisisobutyronitrile $\left(1 \mathrm{~mol} \%\right.$ to total monomers) at $60^{\circ} \mathrm{C}$ for $24 \mathrm{~h}$

\footnotetext{
${ }^{\dagger}$ To whom correspondence should be addressed (akashi@chem.eng.osaka-u.ac.jp).

${ }^{\dagger \dagger}$ Present Address: Research Center for Advanced Science and Technology, The University of Tokyo, 4-6-1 Komaba, Meguro-ku, Tokyo 1538904, Japan

${ }^{\dagger \dagger}$ Present Address: Department of Molecular Chemistry, Osaka University, 2-1, Yamada-oka, Suita 565-0871, Japan
} 
Table I. Preparation and characterization of corecorona nanospheres

\begin{tabular}{lrcccc}
\hline Macromonomer & $M_{\mathrm{n}}$ & $M_{\mathrm{w}} / M_{\mathrm{n}}$ & $\begin{array}{c}\text { St/Macro. } \\
(\mathrm{mol} / \mathrm{mol})\end{array}$ & $\begin{array}{c}\text { Particle size }^{\mathrm{a}} \\
(\mathrm{nm})\end{array}$ & $\begin{array}{c}\text { C.V. }^{\mathrm{b}} \\
(\%)\end{array}$ \\
\hline PMAA & 2700 & 1.8 & 30 & 740 & 11 \\
& 7500 & 2.4 & 35 & 440 & 17 \\
& 7500 & 2.4 & 85 & 680 & 16 \\
& 7500 & 2.4 & 110 & 760 & 17 \\
& 10300 & 2.5 & 160 & 730 & 11 \\
PNIPAAm & 3000 & 2.8 & 50 & 690 & 13 \\
& 3000 & 2.8 & 80 & 870 & 15 \\
& 3000 & 2.8 & 120 & 1060 & 17 \\
\hline
\end{tabular}

${ }^{\text {a }}$ Particle size analyzed by DLS. ${ }^{\mathrm{b}}$ Coefficient of variation estimated from [(standard deviation of particle size)/(particle size) $] \times 100$.

of polymerization time. The total amount of monomers was fixed to $1 \mathrm{~g}$. After purification by three times centrifugation using water, PSt nanospheres with both PMAA and PNIPAAm corona dispersed in water (respective solution $\mathrm{pH}$ of 2 and 6) were obtained. Preparative conditions and particle size data at $20^{\circ} \mathrm{C}$ are shown in Table I. Particle size was changed by changing the monomeric ratio for copolymerization using the same macromonomer. To obtain similar size using macromonomers with different molecular weights, monomer ratios were suitably changed. ${ }^{9,10}$ The size in the nanospheres was analyzed by dynamic light scattering (DLS) (Coulter model N4SD) or from conventional TEM images for intact nanospheres without cutting (diameters were measured with a ruler). At least ten particles were measured for the latter analysis, and then mean diameters and size distribution were obtained. For TEM (Hitachi H700) observation, nanosphere dispersion was cast on a copper mesh and dried under reduced pressure, and subsequently carbon was sputtered with $20-50 \mathrm{~nm}$ thickness.

\section{Analysis of Cross-Sections}

The preparation of cross-sections was basically reported in a previous paper. ${ }^{22}$ Briefly, $0.01 \mathrm{~g}$ of lyophilized nanospheres with PMAA corona layers were quickly dispersed in a mixture of ERL4206 $(0.5 \mathrm{~g})$, Quetol-653 (0.86g), and NSA (3.3 g), and subsequently, S-1 $(0.05 \mathrm{~g})$ was added to obtain nanosphere-imbedded resin. For nanospheres with PNIPAAm corona, S-1 was changed to DER736. All of these chemicals were purchased from Nisshin EM (Japan). The dispersed solutions were hardened for $2 \mathrm{~d}$ at $60^{\circ} \mathrm{C}$. By setting the nanosphere embedded resin to a microtome (LKB 8800 ULTROTOME III, Sweden) with a glass knife, we obtained cross-sections with less than around $0.1 \mu \mathrm{m}$ thickness with a cutting rate
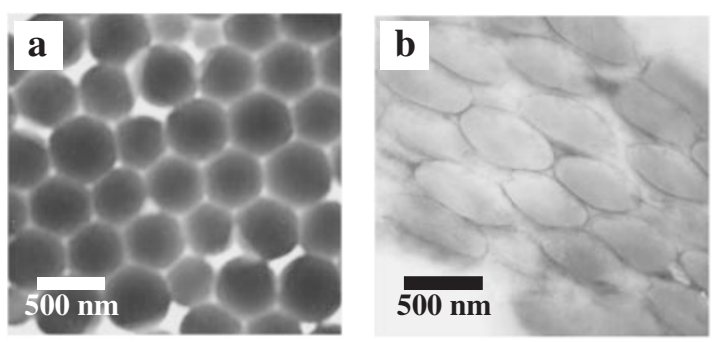

Figure 1. TEM images of (a) intact PSt nanospheres with PMAA $\left(M_{\mathrm{n}} 7500\right)$ corona layers prepared from St/macromonomer ratio, 35/1 (mol/mol) and (b) stained nanosphere cross-sections.

of $2 \mathrm{~mm} \mathrm{~s}^{-1}$ on a water surface. Sections on a water surface were put on a copper mesh and dried under reduced pressure. Sections were exposed to a vapor of osmium tetraoxide for $20 \mathrm{~min}$ at $25^{\circ} \mathrm{C}$. The conditions for the preparation of stained cross-sections were optimized. Stained sections were observed by TEM (Hitachi H700) with an accelerate voltage of 200 $\mathrm{kV}$. To measure the thickness of corona layers from cross-sections, nanospheres having a similar size to normal TEM images were selected to obtain appropriate corona thickness (otherwise, the thickness would be a higher value).

\section{RESULTS AND DISCUSSION}

Typical TEM images of cast nanospheres and their cross-sections are shown in Figure 1. Nanospheres were spherical in form, and their size distributions were narrow (Figure 1a). Nanospheres with different corona compositions, different corona molecular weights, and with different sizes were prepared, as shown in Table I. In TEM images of cross-sections (Figure 1b), well-stained rims, which corresponded to corona layers, were observed, similar to our previous study. ${ }^{22}$ Both the PMAA and PNIPAAm corona layers were more heavily stained than PSt cores, possibly due to the fact that osmium tetraoxide favorably interacts with respective carboxyl and amide groups. Such rims were not observed for stained cross-sections of commercially available polystyrene latex particles. These observations indicate that the present nanospheres also have core-corona structures. Since nanospheres were slightly compressed during the cutting process, the corona layer thickness was estimated by measuring the thickness at a long axis, as cited in our previous study. ${ }^{22}$

Figure 2 shows dependence of both corona layer thickness and particle size (measured from TEM images) against the feed molar ratio of St and PMAA macromonomer $\left(M_{\mathrm{n}} 7500\right)(\mathrm{St} / \mathrm{macromonomer}$, mol/ $\mathrm{mol})$. When the same macromonomer was used, corona layer thickness was almost the same in all cases, 


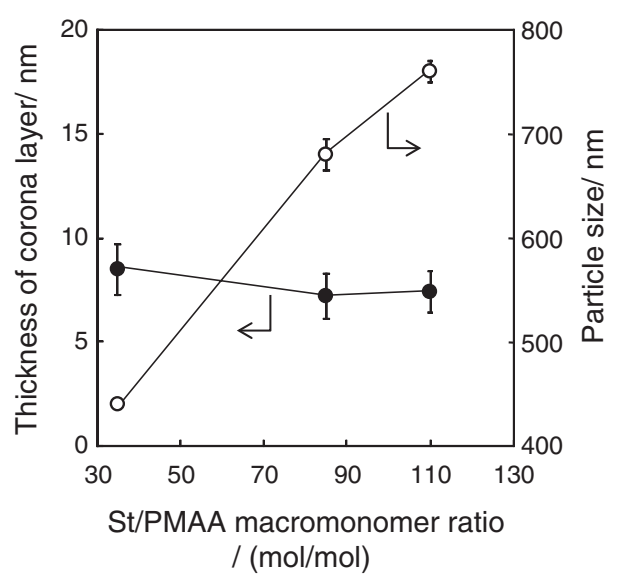

Figure 2. PSt nanospheres with PMAA coronas show dependence of both the corona layer thickness and the particle size against molar ratios of St and macromonomer $\left(M_{\mathrm{n}} 7500\right)$.

even though particle size increased with increasing molar ratio. This observation indicates that corona layer thickness was independent of particle size, and was dependent on the macromonomer used for copolymerization with St. Mean thickness was estimated to be $7-8 \mathrm{~nm}$. Assuming a fully extended trans-zigzag macromonomer conformation, length can be calculated to be approximately $13 \mathrm{~nm}$. This estimation suggests that corona layers are comprised of monolayers derived from macromonomers, and that the surface polymer component has coiled conformation. Figure 3 shows the dependence of both corona layer thickness and particle size (measured from TEM images) against macromonomer $M_{\mathrm{n}}$. Although nanospheres with similar particle size were used, corona layer thickness clearly increased with increasing $M_{\mathrm{n}}$. Assuming a trans-zigzag conformation, length can be estimated to be approximately 5,13 , and $18 \mathrm{~nm}$ for macromonomers $\left(M_{\mathrm{n}} 2700,7500\right.$, and 10300).

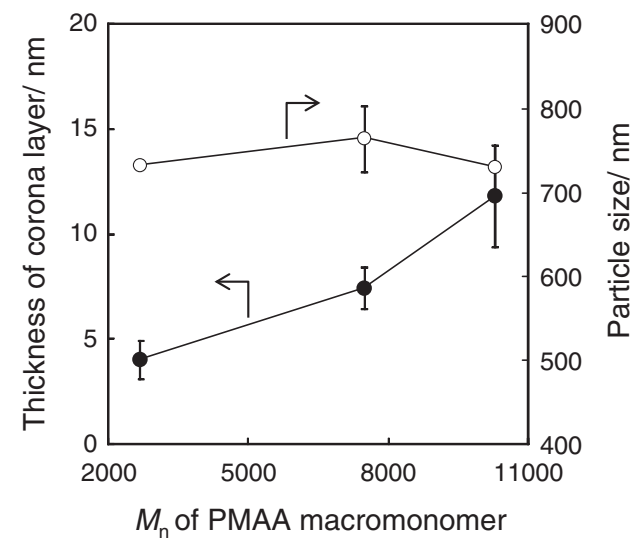

Figure 3. PSt nanospheres with PMAA coronas show dependence of both the corona layer thickness and the particle size against macromonomer $M_{\mathrm{n}}$.
The corona layer thickness analyzed was slightly smaller than the estimated length, except for the case of the macromonomer with $M_{\mathrm{n}} 2700$, also indicating monolayer coverage of nanosphere surfaces with hydrophilic polymers derived from macromonomers. Smaller macromonomers seemed to have more extended conformations on their surfaces. These observations are similar to cross-sectional analyses of PSt nanospheres with PEG coronas, ${ }^{22}$ suggesting that both macromonomers similarly stabilize the surface of PSt nanospheres during copolymerization. It is also concluded that core-corona structures can be observed not only with PEG macromonomers but with PMAA as well, suggesting that core-corona structures are generally generated by a macromonomer method. It is noted that corona layer thickness remained unchanged, even though $\mathrm{pH}$ of the nanosphere solution was changed to 8 using sodium hydroxide (above the $\mathrm{p} K_{\mathrm{a}}$ of PMAA). This observation also suggests that two-dimensionally well-packed PMAA chains exist on nanosphere surfaces.

It is well known that PNIAAm has a LCST at approximately $32{ }^{\circ} \mathrm{C}$, followed by a coil-globule transition. ${ }^{24}$ In the case of PSt nanospheres with PNIPAAm coronas, particle size was decreased above the LCST, and reversibly increased below this temperature. ${ }^{23}$ However, change in diameter analyzed by DLS was much greater than estimated length of PNIPAAm macromonomers. In fact, $\Delta$ (diameters) were more than $50 \mathrm{~nm}$ even in cases of macromonomers with several thousands $M_{\mathrm{n}} \cdot{ }^{23}$ To interpret the above thermoresponsive process, cross-sections were also analyzed. Figure 4 shows the dependence of both corona layer thickness and particle size (measured from TEM images) against the feed molar ratio of St and PNIPAAm macromonomers $\left(M_{\mathrm{n}} 3000\right)$ (St/macro-

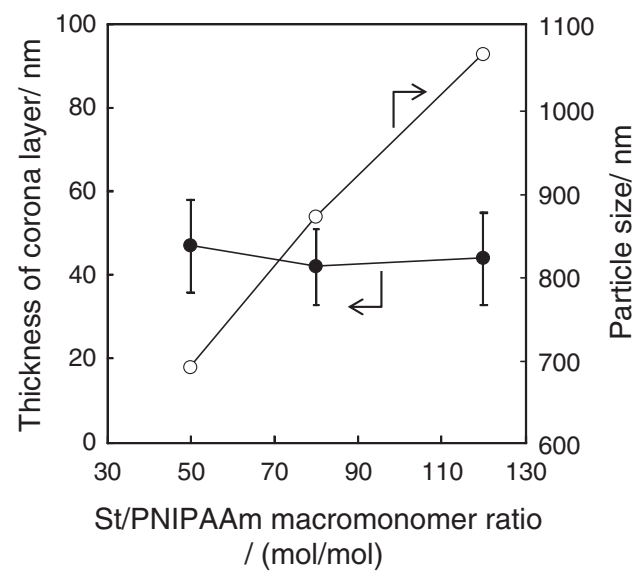

Figure 4. PSt nanospheres with PNIPAAm coronas show dependence of both the corona layer thickness and the particle size against molar ratios of St and macromonomer $\left(M_{\mathrm{n}} 3000\right)$. 
monomer, $\mathrm{mol} / \mathrm{mol}$ ). Corona layer thickness was the same within experimental error, although particle size increased with increasing ratios. These observations were consistent with results obtained from other nanospheres. However, mean corona layer thickness was estimated to be approximately $44 \mathrm{~nm}$, and was much greater than the fully extended length of macromonomers and those of other nanospheres (it is noted that corona layers are possibly comprised of macromonomer-rich graft-copolymers). Furthermore, the $\Delta$ (diameters) of present nanospheres, analyzed between 20 and $38^{\circ} \mathrm{C}$ (measured by DLS), were $150-430 \mathrm{~nm}$, and increased with increasing particle size, similar to results already reported. ${ }^{23}$ Accordingly, it was confirmed that PNIPAAm corona layers as well as PSt cores simultaneously shrank above a LCST. This is possibly due to partial mixing of PSt and PNIPAAm within the core. These observations also suggest that surface stabilization of PNIPAAm was clearly different from that of other macromonomers. As a consequence, internal structures of nanospheres seem to be affected by chemical structures of macromonomers.

Considering that the feed wt $\%$ of macromonomers was as high as $67 \mathrm{wt} \%$ for the nanosphere prepared using PMAA macromonomer $\left(M_{\mathrm{n}} 7500, \mathrm{St} /\right.$ macromonomer molar ratio 35) (see Table I), the volume fraction of corona layers analyzed is much smaller than expected values. Previous study demonstrated the sufficient reactivity for copolymerization between St and $p$-chloromethylstyrene-terminated macromomers. ${ }^{6,9,10}$ On the other hand, differential scanning calorimetric study of PSt nanospheres with PEG coronas demonstrated melting peaks of PEG crystals presented in nanosphere cores. ${ }^{25}$ These observations suggest that PMAA and PNIPAAm segments were also located in cores. Hydrophilic branches of graft copolymers seem to be partially incorporated in cores during copolymerization.

Cross-sectional morphologies of polymeric nanospheres prepared with two macromonomers were analyzed by TEM. Typical core-corona structures were clearly observed for all nanospheres, indicating that the macromonomer method can generally synthesize nanospheres with unique structures. Corona layers were basically comprised of polymer monolayers derived from macromonomers, although structures seemed to be affected by macromonomer chemical species. The present knowledge is important to further utilize core-corona nanospheres and to understand various biomedical nanosphere functions.

Acknowledgment. We would like to acknowledge
Dr. K. Arai, Kagoshima University, Japan, for the use of microtome.

\section{REFERENCES}

1. M. Akashi, I. Kirikihira, and N. Miyauchi, Angew. Makromol. Chem., 132, 81 (1985).

2. M. Akashi, T. Yanagi, E. Yashima, and N. Miyauchi, J. Polym. Sci., Part A: Polym. Chem., 27, 3521 (1989).

3. M. Akashi, D. Chao, E. Yashima, and N. Miyauchi, J. Appl. Polym. Sci., 39, 2027 (1990).

4. M. Riza, S. Tokura, A. Kishida, and M. Akashi, New Polym. Mater., 4, 189 (1994).

5. T. Serizawa, K. Taniguchi, and M. Akashi, Colloids Surf., 169, 95 (2000).

6. M. Riza, S. Tokura, M. Iwazaki, E. Yashima, A. Kishida, and M. Akashi, J. Polym. Sci., Part A: Polym. Chem., 33, 1219 (1995).

7. T. Serizawa, M. Q. Chen, and M. Akashi, J. Polym. Sci., Part A: Polym. Chem., 36, 2581 (1998).

8. M. Q. Chen, A. Kishida, and M. Akashi, J. Polym. Sci., Part A: Polym. Chem., 34, 2213 (1996).

9. M. Q. Chen, T. Serizawa, A. Kishida, and M. Akashi, J. Polym. Sci., Part A: Polym. Chem., 37, 2155 (1999).

10. M. Q. Chen, T. Serizawa, and M. Akashi, Polym. Adv. Technol., 10, 120 (1999).

11. S. Kawaguchi, K. Ito, and M. A. Winnik, Macromolecules, 28, 1159 (1995).

12. K. Ishizu and N. Tahara, Polymer, 37, 1729 (1997).

13. A. J. Paine, W. Luymes, and J. McNulty, Macromolecules, 23, 3104 (1990).

14. S. Takeuchi, M. Okie, C. Kowitz, C. Shimasaki, K. Hasegawa, and H. Kitano, Makromol. Chem., 194, 551, (1993).

15. K. Sugiyama, K. Shiraishi, K. Ohga, H. Shirahama, H. Tamai, K. Kikukawa, and H. Yasuda, Polym. J., 25, 521 (1993).

16. S. Kobayashi, H. Uyama, S. W. Lee, and Y. Matsumoto, J. Polym. Sci., Part A: Polym. Chem., 31, 3133 (1993).

17. J. Liu, C. H. Chew, L. M. Gan, W. K. Teo, and L. H. Gan, Langmuir, 13, 4988 (1997).

18. M. Akashi, T. Niikawa, T. Serizawa, T. Hayakawa, and M. Baba, Bioconjugate Chem., 9, 50 (1998).

19. S. Sakuma, N. Suzuki, H. Kikuchi, K. Hiwatari, K. Arikawa, A. Kishida, and M. Akashi, Int. J. Pharm., 149, 93 (1997).

20. T. Serizawa, T. Uchida, and M. Akashi, J. Biomater. Sci., Polym. Ed., 10, 391 (1999).

21. C. W. Chen, M. Q. Chen, T. Serizawa, and M. Akashi, Chem. Commun., 831 (1998).

22. T. Serizawa, A. Takehara, and M. Akashi, Macromolecules, 33, 1759 (2000).

23. M. Q. Chen, T. Serizawa, M. Li, C. Wu, and M. Akashi, Polym. J., 35, 901 (2003).

24. S. Fujishige, K. Kubota, and I. Ando, J. Phys. Chem., 93, 3311 (1989).

25. K.-I. Hiwatari, T. Serizawa, A. Kishida, and M. Akashi, Polym. J., 33, 424 (2001). 